June 25 - June 30, 2019, San Diego, USA

https://doi.org/10.1007/978-3-030-23404-1_6

\title{
Blockchain Interoperable Digital Objects
}

\author{
Babu Pillai $^{1}$, Kamanashis Biswas ${ }^{1,2}$ and Vallipuram Muthukkumarasamy ${ }^{1}$ \\ ${ }^{1}$ Griffith University, Gold Coast, Australia. ${ }^{2}$ Australian Catholic University, Sydney, Australia. \\ babu.pillai@griffithuni.edu.au, kamanashis.biswaseacu.edu.au, \\ v.muthulgriffith.edu.au
}

\begin{abstract}
The future of distributed ledger technology such as blockchain is dependent on its ability to interact and integrate with other systems. Therefore, interoperability has become a fundamental issue that needs to be addressed. The emerging category of crypto-assets are managed and understood using different frameworks. There is, therefore, a need for a unified classification of crypto-assets. This work aims to bring some clarity to and understanding on interoperable crypto-assets and their characteristics. This paper categorizes digital crypto-assets for the purpose of implementing interoperability. The categorization of crypto-assets is based on their functionalities and their purpose. An interoperability scenario has been given for the defined crypto-asset classes.
\end{abstract}

Keywords: Blockchain, Distributed Ledger Technology, Interoperability, Digital Assets, Crypto-assets, Crypto-coins, Crypto-tokens.

\section{Introduction}

The blockchain technology has emerged as a disruptive technology, that enable trust among untrusted network nodes in the digital world. Blockchain the underlying technology behind bitcoin has enormous potential for enhancing the trustworthiness of data in a distributed environment, [1] and is foreseen as a possible solution to a number of challenging problems across many domains [2]. The technology adds trustable value to digital entities in the Internet domain and makes it possible to transfer value, rather than information over the network [3,4]. These unique characteristics open a new form of cryptographic assets, which have generated significant interest in another type of digital assets. With the promise of trustability, transparency, and traceability of digital objects registered in the system, the blockchain technology has attracted significant research studies and industrial attention [5].

A diverse ecosystem of blockchain projects with different protocols and cryptographic structures offering a variety of solutions has emerged to serve the needs of the digital world. However, these blockchains remain isolated, operating in their own respective silos, each one with its own ecosystem, consensus model, and network. Many of these projects offer a solution to a specific problem [6] such as the decentralized 
marketplace ${ }^{1}$, and open-bazaar ${ }^{2}$. Thus, it has become clear that there will exist many more independent networks of blockchains designed for specific problems. The applications developed on these networks need to cross communicate with each other to provide real services in a broad range of situations. This emerges as a new paradigm of "establishing connections between isolated blockchain networks" creating the concept of interoperability [4].

Interoperability is generally referred to as the ability of different systems to communicate with each other in a distributed environment to exchange or retrieve information/data. The application that operates on each system must interpret the data and understand the meaning of the exchanged information. In the case of blockchain based system, the most significant obstacle to be overcome in the creation of this interoperable network of blockchains would be the preservation of what makes each chain unique when the value moves from one chain to the other. A recent study conducted by Hileman and Rauchs [7] suggests that "Interoperability will be essential for the massive adoption of blockchain and distributed ledgers." Therefore, whether a public or private type of blockchain, interoperability across blockchain systems will become a core requirement [8].

The concept of interoperability among blockchain based systems is not fully understood and it is vital for the future growth of the blockchain industry. It has been seen that interoperability solutions are viewed from the perspective of a generalized information system $[9,10]$. However, this perspective of interoperability has been failed to achieve true and adequate levels of interoperability for every situation. Hence, a concept of a systematically categorized perspective is needed. Therefore, we need to look at the design philosophy of this technology and categorize the type of digital cryptoassets the system is holding that require interoperability. Thus, there is a clear requirement for a unified classification of interoperable asset classes. The purpose of this classification is to provide an independent type of crypto-assets in order to guide the design and implementation of interoperability. In this paper, we propose a classification of crypto-assets, based on the purpose and the type of value the asset carries, which helps to understand the crypto-asset landscape.

The rest of the paper is structured as follows. Section 2 introduces interoperability, its challenges, goals, approaches and mechanisms. Section 3 discusses blockchain and digital objects classifications. Section 4 describes a crypto-asset classification for blockchain based systems. In Section 5, we formalize the classification and map the crypto-asset class with an interoperability scenario, and finally, Section 6 concludes the paper.

\section{Interoperability}

Interoperability refers to the ability of two or more systems to provide service or accept service from the other system and to utilize the service of a common exchange effectively together [11]. The linkage should allow these connected systems to exchange data accurately, effectively, and consistently [12]. That means the application

\footnotetext{
$1 \mathrm{https} / / /$ coincentral.com/decentralized-marketplace-blockchain/

2 https://openbazaar.org/
} 
that operates on each system must understand the functionality, which is available for the other system. Software level interoperability is essential since it allows information to be shared without an intermediary. Furthermore, a common standard will enhance the possibility of in-built interoperability $[4,13]$.

For a blockchain technology-based system, interoperability refers to cross-communication between different blockchains that enables to exchange or retrieve information or values. This deals with information obtained from another system and makes a change to the state of that system based on the received information. However, inherently the blockchain is an 'append only' model, and the state can only be appended through transactions, by nodes within its own network using their consensus mechanism [1, 13-16]. Therefore, here the underlying assumption is that "cross-communication is not intended to make direct state changes to another blockchain system. Instead, a cross-communication should trigger some set of functionalities on the other system that expected to perform an operation within its own network", as an example, verifying the authenticity of information requested within its own network. However, for the process of interoperability cross-communication remains a challenge - because interoperability requires the integration of different interlinked information sources [17].

\subsection{Challenges}

Considering the Internet or intranet as an overall network and blockchain as platforms with sub-networks within it holds a variety of digital assets. The current state-ofthe-art blockchain technology is architectured in such a way that it operates as a standalone system. It is designed so that a network of node participants, who are the stakeholders, decide on the current state of the system based on an agreed protocol [15]. This protocol dictates the value and the consensus model. Most importantly the value has been created by and exists only within the system and its nodes [13]. This means, enabling interoperability is a way to exchange value from one blockchain system to another system. However, validating data from another system is challenging because each systems' value is unique and no cross-chain standard classifying crypto-assets' value exists. Therefore, each blockchain system has its own idiosyncratic interoperability issues that cannot be addressed using a general information systems perspective. This is the main challenge we address so that data and digital assets can be exchanged between sub-networks.

\subsection{Goals of Interoperability}

Generally, interoperability is developed through functional design principles and standards thus it forms a base for different applications to communicate and helps to automate the process. Many approaches have been proposed to achieve interoperability such as: Integrated approach - where a commonly agreed format of data structure exists; the Unified approach - where a common format with semantic understanding exists; and the Federated approach - where connections established accordingly [18]. A common goal of the interoperability approach is to enable cross-communication using different types of technologies. 
Interoperability seems to be a strategical concept, where different systems crosscommunicate to achieve a common goal. Here the desired goal is to connect separate networks of ledger systems and facilitate cross-chain communication in order to interact and transfer data. To understand the interoperability goal, it is first necessary to identify the scenarios for interoperability: an active mode - where systems must be able to engage in the interaction to send and receive data; and a passive mode where systems able to receive data [4]. Therefore, the desired interoperability generally falls into two categories, identified as:

- Cross-chain 'transfer \exchange' - a cross-chain transfer process aims to transfer various types of assets or value from one blockchain system to another. That means the systems must be in active mode and have a common understanding of the semantics so that the transfer occurs meaningfully.

- Cross-chain 'validation \verification' - a cross-communication process aims to provide the ability to verify assets, value or information between the blockchain systems.

In order to facilitate cross-communication, many techniques such as sidechain, relay, notary schemes and hash-locking are under development [19]. A variety of approaches have been proposed to achieve interoperability [4, 8], but nearly all of them lead to the violation of the principles of decentralization. The core benefit of a blockchain technology-based system is to overcome the risk of centralization.

\subsection{Approaches for Interoperability}

Interoperability approaches aim to address interoperability barriers however, we must consider how these barriers are removed [18] because some approaches may lead the system to change its security model. Considering the decentralized nature of the architecture, where multiple nodes participate in the process to reach finality, nodes must retain the same result. For that, nodes must have or be given the information in order to process the transaction. If the nodes are set to fetch data from other blockchain systems, the dynamic nature of values would interfere with the consensus. Therefore, the exchange process must be carefully designed in accordance with the system goal. This leads to the interoperability focuses on two types of approaches: centralized and decentralized.

- In a centralized approach, the cross-communication operation is triggered by a single entity and operates directly between the sender and the receiver blockchain. This results in the cross-communication process in a closed environment. The inner communication is facilitated through some trusted/ credible nodes acting as notaries to verify whether a specific event has happened on one chain and taken agreed action on another chain [4, 19-21].

- A decentralized approach assumes that the cross-communication occurs automatically at a protocol level through smart contracts in a distributed environment. For example, when Bob invokes a transfer transaction on his chain, it will automatically be credited in Alice's chain. 
Current research is experimenting several mechanisms with the aim of achieving interoperability among networks of blockchains.

\subsection{Interoperability Mechanisms}

Many research groups and industries are actively investigating multiple blockchain architectures and protocols that allow blockchains to cross-communicate between different networks and thus facilitate the exchange of transactions. Many FinTech start-up companies are also working on various blockchain architectures and protocols to address interoperability.

Pegged side-chain is an addition to the bitcoin protocol and enables assets to be transferred back and forth between multiple blockchains [22]. Generally, this can be implemented in any blockchain system that holds an asset, token or cryptocurrency. The 'parent' chain, known as the main blockchain is connected by a new blockchain called a side-chain. There is little interaction between the two. The side-chain as the custodian of assets from the parent chain, and this same asset is locked in the main chain to prevent double spending. However, the advantage of this side-chain is that it can perform instant transactions at a higher speed and volume. Micropayments are the most common use case for side-chains. In this system, it is not necessary to record every transaction between two parties on the main blockchain. If only a handful of parties are concerned about a recurring transaction, it is not necessary for all the other nodes to be aware of those transactions. Instead, a direct connection should be created between the two pairs which perform transactions on a recurring basis for a certain period of time and only the final balance is recorded on the blockchain [23].

Relay is a mechanism where a 'Chain $A$ ' actively listens to and keeps a record of part of the information such as block header from another 'Chain $B$ '. This will be useful for a light client to verify block headers belonging to 'Chain $B$ ' by using a standard verification process [19].

Hash-locking is another technique for the exchange of digital assets without a trusted third party. The mechanism utilizes a hash time [24] locked system which puts a time lock on the transaction so that both the obligations are fully met, otherwise the transaction cannot occur - atomic transaction [19].

Bridges or gateways are the intermediate mechanism aim to provide interoperability between systems. The objective here is to bridge the differences between various data standards, and middleware. To perform a conversion between the protocol of the sending system and the protocol of the receiving system, the gateway can be expanded with the use of plug-ins.

\section{Blockchain and Digital Objects}

At their core, blockchains are decentralized databases maintained by a network of computers. Blockchain technology enables the digital representation of assets and their secure transfer of value [25]. By design, the security of the value transfer is guaranteed by the interaction protocol itself and obviates the need for trusted transaction intermediaries [13]. Bitcoin has emerged as the first blockchain application of a decentralized 
crypto-currency system [25]. Even though Bitcoin blockchain was implemented as a decentralized currency system, the application is, in fact, a software system that executes a scripting language in a distributed environment. To think beyond the payment system required new developments in the technology itself which lead to the development of the Ethereum project [26]. Ethereum was developed as a platform that could run programmed applications on blockchain through smart contracts [27]. Thus, it created a wide variety of decentralized applications which opened the technology to the possibility of digital assets and tokens [28-30]. With the ability to tokenize and decentralize not only cryptocurrency but also other scarce assets the blockchain technology significantly expanded its disruptive potential [29].

Blockchain technology offers a verifiable way to track digital transactions. This makes this technology useful for digital asset management systems. Such functionality offers the storage and transacting of crypto-assets [25]. This is a use case where a system holds a crypto-asset and the user will be able to transfer the asset between systems. Blockchain also allows crypto-assets to be distributed while protecting them from being copied. Thus, the technology is useful to track assets as they move through the systems in a distributed environment [31]. The advantage of a low transaction fee and not having to rely on a single entity are the main benefits of this technology [13].

\subsection{Digital Object Classification}

Digital objects [32] are an essential part of a modern information system that strives towards technology-independent and future-proof automated operations between software and computer systems. Digital objects exist solely in the digital space and carry a state of information [32]. Further, they can be classified into different digital asset classes to fully bridge the gap between physical and digital mixed world.

Primarily, there are two ways of representing digital objects, tangible and intangible objects. Tangible items are classified as objects with physical existence, such as car, house, and they are unique. In the context of the blockchain, a tangible object represents an asset which has a physical existence as well represented in a digital form. The classification of asset objects is based on the tangibility of the assets. Intangible items are those items that do not have a physical nature such as service and are represented as abstract objects within the system. Further, as referred in Table 1, within the type of the tangible and intangible objects, there are 'fungible' and 'non-fungible' objects [33].

Fungible objects belong to a digital object class which are exchangeable and are built using a common standard, value and characteristics, such as currency and ERC $3-20^{4}$ tokens. Cryptocurrencies are perfect examples of fungible tokens, in fact, fungibility is the essential feature of any currency. However, if we take the fungibility out of it, then it becomes a non-fungible token, which is a unique, non-interchangeable special type of objects, such as a birth certificate, passport and ERC- $21^{5}$ tokens.

\footnotetext{
Ethereum Request for Comments

4 https://theethereum.wiki/w/index.php/ERC20_Token_Standard

$5 \mathrm{https} / / /$ medium.com/crypto-currently/the-anatomy-of-erc721-e9db77abfc24
} 
Table 1. Characteristics of assets

\begin{tabular}{lll}
\hline Characteristics & Definition & Examples \\
\hline Tangible & $\begin{array}{l}\text { Has a physical existence. } \\
\text { Intangible }\end{array}$ & Land, Property. \\
things. & Services, ID. \\
Fungible & Built using a common standard. & $\begin{array}{l}\text { ERC-20 tokens, } \\
\text { Currency. }\end{array}$ \\
Non-fungible & Type of objects that are unique. & $\begin{array}{l}\text { Birth certificate, } \\
\text { Passport. }\end{array}$ \\
\hline
\end{tabular}

\section{Crypto-assets}

The generic definition of an asset is a resource which an individual or organization owns or controls and which is expected to produce future economic value. Assets used to be classified as tangible objects, such as buildings, and intangible objects such as intellectual property [34]. The proliferation of digital technology has created a new class of assets known as Data Assets or Digital Assets which exist in binary format, examples of which are digital pictures and Facebook accounts. In the context of this paper, we refer asset as a digital representation of an item that is being created and exists in a blockchain.

Blockchain technology and its services have given birth to a new cryptographic form of assets termed as crypto-assets [29, 35]. Crypto-assets are a type of digital assets, recorded on a blockchain ledger, which utilize techniques such as cryptography, distributed consensus, peer-to-peer network, and smart contract [36] in order to create, transact and verify in a decentralized manner [30, 37], such as BTC and ETH [17]. They derive their names from the cryptographic security mechanisms used within the distributed systems.

The concept of crypto-asset [14] in blockchain systems is essentially a technology that produces virtual tokens that represent value in a closed network. The primary weakness of such crypto-asset token-based systems is its inability to operate outside of its network. Currently, it is being facilitated through third-party intermediaries. Cryptoassets are also referred to as crypto-tokens or crypto-coins, which are primarily based on the asset's functionality. In the context of this paper, 'coin' refers to a cryptographic asset used as a medium of value exchange, whereas the term 'token' refers to an abstract category of digital assets, that acquires specific features depending on the context.

The primary purpose of these crypto-assets is to be used as a medium of exchange independent of any central bank, and with a specific value [37, 38], such as currency, a place holder for digital representation of objects and services. There are different classifications of these assets based on their functionalities and purposes [30, 39]. There are frequent discussions on whether the crypto-assets can be classified as money or assets [37, 40]. However, this paper does not cover the legal or accounting sides of crypto-assets. 
Crypto-assets and their taxonomy are arguably the most important component for enabling interoperability in the blockchain space. Many forms of crypto-assets exist however when you separate them based on the type and functionality most crypto- assets fall into one of the following categories as shown in Table 2.

Table 2. Classification of crypto-assets

\begin{tabular}{ll}
\hline Asset Class & Definition \\
\hline Crypto-coin & $\begin{array}{l}\text { Representation of digital objects that express the purpose of } \\
\text { acting as a medium of exchange or unit of account and imple- } \\
\text { mented at a protocol level, for example, BTC, ETH. }\end{array}$ \\
\hline Asset-token & $\begin{array}{l}\text { Representation of an object that has some characteristics, for } \\
\text { example, a car, property. }\end{array}$ \\
\hline Utility-token & $\begin{array}{l}\text { Representation of digital objects that provide the right to ac- } \\
\text { cess or utilize the value derived from it, for example, a ser- } \\
\text { vice or subscription. }\end{array}$ \\
\hline
\end{tabular}

The Fig. 1 represents the relationship between the existing category of assets and the classification of blockchain crypto-assets. For example, an asset-token can represent a tangible or intangible item, however, a crypto-coin represents an intangible object. Each of these crypto-assets can be considered 'digital objects' with their own individual properties.

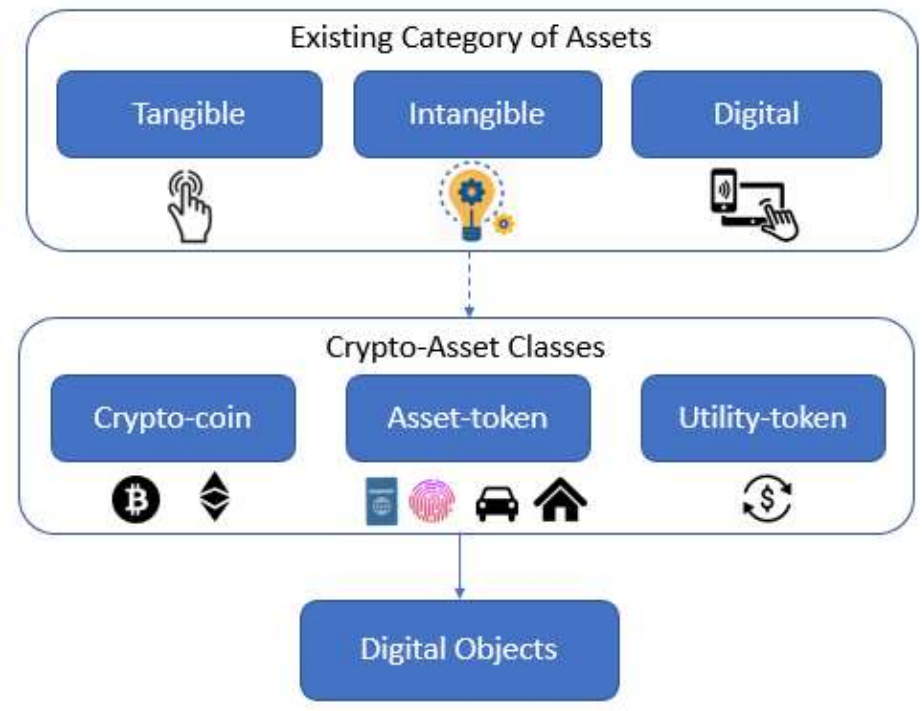

Fig. 1. Blockchain crypto-asset classes

In the blockchain space, crypto-asset is a new concept. To the best of our knowledge, there is no generally accepted standard for asset classification in the crypto space. Therefore, apart from these three basic categories shown in Table 2, there is much potential for the creation of new asset classes because crypto-assets are digital 
representation of objects. These objects achieve shape and inherit characteristics when they are mapped to an appropriate digital object. A brief description of the given asset class and their properties are described in the following subsection.

\subsection{Crypto-coins}

Crypto-coins are also referred as crypto-currencies, a new form of money, implemented on the blockchain for the purpose of a medium of exchange independent of any central control such as a bank. Crypto-currencies such as BTC or ETH are called native currencies. Because they are developed for and exist within the system and are used to pay for the computational service offered by the system. These are also used as paymentcurrency where payments can be made for goods or services [41]. Crypto-coins such as BTC and ETH are built into the system as part of the protocol. Therefore, they are not directly exchangeable between other systems, instead they can only be traded.

\subsection{Asset-tokens}

Unlike crypto-coin, asset-tokens are not native to a blockchain they are created on top of a blockchain and can be used to represent a wide range of assets beyond currencies. Asset tokens are commonly implemented in the smart contracts that may have physical existence such as car, property or may be without a physical existence such as company shares. The domain of digital technology including supply chain is increasingly dependent on the effective management of digital assets which have been managed by central entities [42]. However, these entities have used proprietary techniques which are usually slow, costly, insecure and vulnerable to abuse. Blockchain would be an effective solution to manage digital assets more effectively. CryptoKitties ${ }^{6}$ are a classical example of non-fungible tokens that are digital collectables and unique to each other. Some other use cases are Know Your Customer ID $^{7}$ for digital academic certificates and copyright [43], supply chain tracking, software licenses, and more.

\subsection{Utility-tokens}

Utility tokens are a type of system or network, distinct digital token that represents a unit of product or service. They are also presented as tokens that enable future access to a product or service [44]. Utility tokens are not designed for investment [45]; rather they are designed to be used as a service which can be purchased. In the blockchain space, ERC20 compatible tokens on the Ethereum platform are considered utility tokens. Other utility-tokens such as TRC10 and TRC $20^{8}$ also exist.

\footnotetext{
https://www.cryptokitties.co/

https://home.kpmg/ie/en/home/insights/2018/02/blockchain-kyc-utility-fs.html

8 https://tron.network/
} 


\section{$5 \quad$ Mapping of Crypto-Assets}

Irrelevant of varies category of assets, digital objects are represented in the same form. However, when we map with a particular type of asset class (crypto-coin, asset-token or utility-token), the digital object gets its form and inherits the characteristics of the assets. Therefore, it is essential to determine and understand the appropriate asset class to represent objects in an interoperable environment. Based on the characteristics (tangible, intangible, fungible and non-fungible) and classification (crypto-coin, asset-token and utility-token) of crypto-assets, we propose a crypto-asset classification framework as shown in Fig. 2.

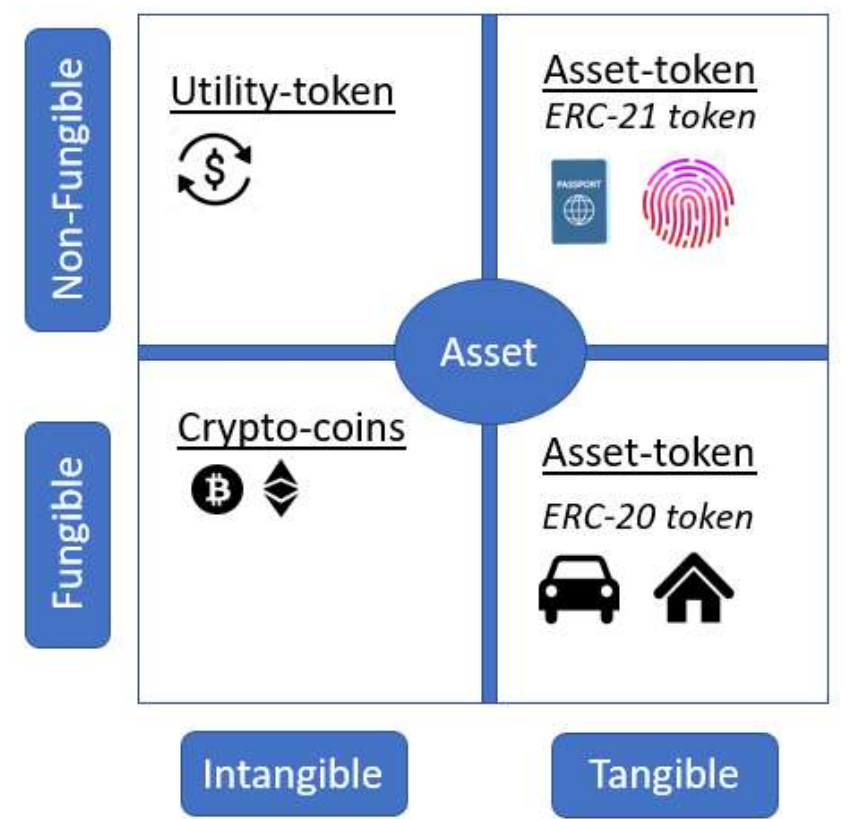

Fig. 2. Crypto-asset characteristics framework

\subsection{Scenario: Crypto-coin}

Generally, crypto-coins are divisible and intangible in nature, unless they have been assigned to different purposes or combinations for a specific purpose. Crypto-coins are formed and exist in a ledger of a distributed system in the form of transaction. Each distributed system runs its own independent ledger and has coin native to it. Therefore, validating one coin from another system is challenging. Currently third-party exchanges facilitating the exchange who has access/share on both the network and act as a liquidity provider facilitating the swapping [40]. There two types of exchanges exist: centralized and decentralized. A centralized exchange dependent on a third-party or intermediary to hold the coin and process the exchange. They offer to swap of a variety 
of crypto-coins mainly through exchange them against fiat currency. Therefore, the centralized exchange is often expensive, inefficient and vulnerable to attack whereas decentralized exchanges do not rely on a third-party service to facilitate the swap. Instead, it occurs directly between users (peer-to-peer) through an automated process. Such a system can be established through a decentralized network using multi-signature, hatch-lock and other solutions. A mapping of crypto-coin with the interoperability approach of centralized and decentralized scenario has been given in Table 3.

Table 3. Crypto-coin interoperability scenario

\begin{tabular}{|l|l|l|l|}
\hline \multicolumn{1}{|c|}{ Crypto-coins } & Centralized approach & $\begin{array}{l}\text { Decentralized ap- } \\
\text { proach }\end{array}$ & Proposed direction \\
\hline $\begin{array}{l}\text { Cross-chain } \\
\text { transfer of } \\
\text { crypto-coins }\end{array}$ & $\begin{array}{l}\text { Through centralized } \\
\text { exchanges. }\end{array}$ & $\begin{array}{l}\text { Through decen- } \\
\text { tralized exchanges } \\
\text { using a mecha- } \\
\text { nism such as } \\
\text { multi-signature } \\
\text { and hash-lock. }\end{array}$ & $\begin{array}{l}\text { If two systems } \\
\text { operate on one } \\
\text { crypto-currency, } \\
\text { then it is a matter } \\
\text { of transferring } \\
\text { from one system } \\
\text { to another in an } \\
\text { agreed way using } \\
\text { gateways. }\end{array}$ \\
\hline $\begin{array}{l}\text { Validation/veri- } \\
\text { fiability }\end{array}$ & $\begin{array}{l}\text { Through centralized } \\
\text { services such as a no- } \\
\text { tary. }\end{array}$ & $\begin{array}{l}\text { Yes, a relay sys- } \\
\text { tem can be imple- } \\
\text { mented to verify } \\
\text { the block. }\end{array}$ & $\begin{array}{l}\text { Web3 and API ac- } \\
\text { cess to verifica- } \\
\text { tion. }\end{array}$ \\
\hline
\end{tabular}

\subsection{Scenario: Asset-token}

Asset-tokens are a digital representation of tangible items in the form of fungible or non-fungible tokens. Each unit of the asset must uniquely identify and hold characteristics based on its asset class, such as, for a car, registration number, model, year of manufacture; and for a property, its ID, land area, land location. Additionally, if the assets are of fungible nature, each unit of the asset must uniquely identify and hold the same characteristics within both systems. An example such as Colored Coin [46] describes a class of methods for representing and managing real-world assets on top of the Bitcoin network. A mapping of Asset-token with the interoperability approach of centralized and decentralized scenario has been given in Table 4 . 
Table 4. Asset-token interoperability scenario

\begin{tabular}{|l|l|l|l|}
\hline \multicolumn{1}{|c|}{ Asset-token } & Centralized approach & $\begin{array}{l}\text { Decentralized ap- } \\
\text { proach }\end{array}$ & Proposed direction \\
\hline $\begin{array}{l}\text { Cross-chain } \\
\text { transfer }\end{array}$ & $\begin{array}{l}\text { Yes, but centralized, } \\
\text { using an intermediary } \\
\text { to process the trans- } \\
\text { fer. }\end{array}$ & $\begin{array}{l}\text { Yes, but the token } \\
\text { that representing } \\
\text { the asset must } \\
\text { have the same se- } \\
\text { mantics. }\end{array}$ & $\begin{array}{l}\text { Common data } \\
\text { standard and } \\
\text { protocol such as } \\
\text { ERC 20 and ERC } \\
\text { 21 tokens. }\end{array}$ \\
\hline $\begin{array}{l}\text { Validation/ veri- } \\
\text { fiability }\end{array}$ & $\begin{array}{l}\text { Centralized valida- } \\
\text { tion through notary. }\end{array}$ & $\begin{array}{l}\text { Yes, a relay sys- } \\
\text { tem to verify the } \\
\text { block data. }\end{array}$ & $\begin{array}{l}\text { Web3 and API } \\
\text { access to } \\
\text { verification. }\end{array}$ \\
\hline
\end{tabular}

\subsection{Scenario: Utility-token}

Utility-tokens are not generally made for exchange purposes; however, there might be a use case where the same product or service may have to exchange information with each other. There is more chance that the exchange may happen between users within the network. Because the token holds the service value, but the network is the one that provides the service. The tokens are designed for spending within a specific blockchain ecosystem. A mapping of Utility-token with the interoperability approach of centralized and decentralized scenario has been given in Table 5 .

Table 5. Utility-token interoperability scenario

\begin{tabular}{|l|l|l|l|}
\hline \multicolumn{1}{|c|}{ Utility-token } & $\begin{array}{l}\text { Centralized ap- } \\
\text { proach }\end{array}$ & $\begin{array}{l}\text { Decentralized ap- } \\
\text { proach }\end{array}$ & Proposed direction \\
\hline $\begin{array}{l}\text { Cross-chain trans- } \\
\text { fer }\end{array}$ & $\begin{array}{l}\text { The value has to be } \\
\text { recreated in the } \\
\text { other system. }\end{array}$ & $\begin{array}{l}\text { Not directly trans- } \\
\text { ferable, it has to } \\
\text { be recreated in the } \\
\text { other system. }\end{array}$ & $\begin{array}{l}\text { Cross-chain } \\
\text { bridge or gate- } \\
\text { ways for burning } \\
\text { the value in one } \\
\text { chain and recreate } \\
\text { the value in an- } \\
\text { other chain. }\end{array}$ \\
\hline $\begin{array}{l}\text { Validation/ verifi- } \\
\text { ability }\end{array}$ & $\begin{array}{l}\text { Centralized valida- } \\
\text { tion through notary. }\end{array}$ & $\begin{array}{l}\text { Can use Cross- } \\
\text { chain bridge and } \\
\text { relay. }\end{array}$ & $\begin{array}{l}\text { Web3 and API } \\
\text { access to } \\
\text { verification. }\end{array}$ \\
\hline
\end{tabular}

Moving forward, it will be vital to distinguish between different cryptographic digital objects that function as crypto-coins, asset-tokens or utility-tokens. A digital cryptoasset can fall into three or more of these categories based on its actual characteristicsand additional categories may not have been invented yet. Therefore, it is difficult to create a lasting category of crypto-assets. However, we assume our classification of crypto-assets will serve as a base for ongoing discussion of current and emerging digital crypto-asset classes. 


\section{Conclusion}

In this paper, we identified the basic category of interoperable crypto-asset classes for blockchain based systems. Further, we provided some clarity on their characteristics and analyzed the current state of interoperability and its proposed directions. Cryptographic assets management is a promising use case for blockchain technology. Adding the concept of interoperability through cross-chain communication enables the transfer of digital assets from one blockchain to another. Assets may be of tangible or intangible in nature, may be implemented at protocol-level or in a smart contract, may be in the form of fungible or non-fungible tokens. Irrespective of the various categories or types, digital objects are represented in the same form as series of binary $1 \mathrm{~s}$ and $0 \mathrm{~s}$. However, when we map with a particular type of asset class, the digital object gets its shape and inherit the characteristics and attributes of the assets. Therefore, it is important to determine and understand the appropriate digital asset class to represent objects. With a clear view of different types of crypto-assets and underlying values, an appropriate interoperability approach can be determined for an expected outcome.

\section{References}

[1] X. Xu et al., "A taxonomy of blockchain-based systems for architecture design," in Software Architecture (ICSA), 2017 IEEE International Conference on, 2017, pp. 243252: IEEE.

[2] K. Peterson, R. Deeduvanu, P. Kanjamala, and K. Boles, "A blockchain-based approach to health information exchange networks," in Proc. NIST Workshop Blockchain Healthcare, 2016, vol. 1, pp. 1-10.

[3] C. P. Chen and C.-Y. Zhang, "Data-intensive applications, challenges, techniques and technologies: A survey on Big Data," Information Sciences, vol. 275, pp. 314-347, 2014.

[4] H. Jin, X. Dai, and J. Xiao, "Towards a Novel Architecture for Enabling Interoperability amongst Multiple Blockchains," in 2018 IEEE 38th International Conference on Distributed Computing Systems (ICDCS), 2018, pp. 1203-1211: IEEE.

[5] G.-H. Hwang, P.-H. Chen, C.-H. Lu, C. Chiu, H.-C. Lin, and A.-J. Jheng, "InfiniteChain: A multi-chain architecture with distributed auditing of sidechains for public blockchains," in International Conference on Blockchain, 2018, pp. 47-60: Springer.

[6] E. Anceaume, A. Del Pozzo, R. Ludinard, M. Potop-Butucaru, and S. TucciPiergiovanni, "Blockchain abstract data type," arXiv preprint arXiv:1802.09877, 2018.

[7] G. Hileman and M. Rauchs, "2017 Global Blockchain Benchmarking Study," 2017.

[8] T. Hardjono, A. Lipton, and A. J. a. p. a. Pentland, "Towards a Design Philosophy for Interoperable Blockchain Systems," 2018.

[9] W. J. Gordon and C. Catalini, "Blockchain Technology for Healthcare: Facilitating the Transition to Patient-Driven Interoperability," Computational and structural biotechnology journal, vol. 16, pp. 224-230, 2018. 
[10] P. Zhang, J. White, D. C. Schmidt, and G. Lenz, "Applying software patterns to address interoperability in blockchain-based healthcare apps," arXiv preprint arXiv:1706.03700, 2017.

[11] F. Vernadat, "Interoperable enterprise systems: architectures and methods," IFAC Proceedings Volumes, vol. 39, no. 3, pp. 13-20, 2006.

[12] A. Geraci et al., IEEE standard computer dictionary: Compilation of IEEE standard computer glossaries. IEEE Press, 1991.

[13] P. Tasca and C. J. Tessone, "Taxonomy of blockchain technologies. Principles of identification and classification," arXiv preprint arXiv:1708.04872, 2017.

[14] I. Alqassem and D. Svetinovic, "Towards reference architecture for cryptocurrencies: Bitcoin architectural analysis," in Internet of Things (iThings), 2014 IEEE International Conference on, and Green Computing and Communications (GreenCom), IEEE and Cyber, Physical and Social Computing (CPSCom), IEEE, 2014, pp. 436-443: IEEE.

[15] Z. Zheng, S. Xie, H. Dai, X. Chen, and H. Wang, "An overview of blockchain technology: Architecture, consensus, and future trends," in Big Data (BigData Congress), 2017 IEEE International Congress on, 2017, pp. 557-564: IEEE.

[16] J. de Kruijff and H. Weigand, "Understanding the blockchain using enterprise ontology," in International Conference on Advanced Information Systems Engineering, 2017, pp. 29-43: Springer.

[17] M. Staples et al., "Risks and opportunities for systems using blockchain and smart contracts. Data61," ed: CSIRO), Sydney, 2017.

[18] D. Chen, "Enterprise Interoperability Framework," in EMOI-INTEROP, 2006.

[19] V. Buterin, "Chain interoperability," R3 Research Paper, 2016.

[20] M. Alipour-Hafezi, A. Horri, A. Shiri, and A. Ghaebi, "Interoperability models in digital libraries: an overview," The Electronic Library, vol. 28, no. 3, pp. 438-452, 2010.

[21] D. Chen, G. Doumeingts, and F. Vernadat, "Architectures for enterprise integration and interoperability: Past, present and future," Computers in industry, vol. 59, no. 7, pp. 647-659, 2008.

[22] A. Back et al., "Enabling blockchain innovations with pegged sidechains," URL: http://www. opensciencereview. com/papers/123/enablingblockchain-innovationswith-pegged-sidechains, 2014.

[23] J. Poon and T. Dryja, "The bitcoin lightning network: Scalable off-chain instant payments," Technical Report (draft), 2015.

[24] M. Herlihy, "Atomic cross-chain swaps," arXiv preprint arXiv:1801.09515, 2018.

[25] J. Rohr and A. Wright, "Blockchain-based token sales, initial coin offerings, and the democratization of public capital markets," Hastings LJ, vol. 70, p. 463, 2018.

[26] G. Wood, "Ethereum: A secure decentralised generalised transaction ledger," Ethereum project yellow paper, vol. 151, pp. 1-32, 2014.

[27] N. Szabo, "The idea of smart contracts," Nick Szabo's Papers and Concise Tutorials, vol. 6, 1997.

[28] F. Knirsch, A. Unterweger, and D. Engel, "Implementing a blockchain from scratch: why, how, and what we learned," EURASIP Journal on Information Security, vol. 2019, no. 1, p. 2, 2019. 
[29] Y. Chen, "Blockchain tokens and the potential democratization of entrepreneurship and innovation," Business Horizons, vol. 61, no. 4, pp. 567-575, 2018.

[30] S. Masnavi, "CryptoCompare Publishes 'Cryptoasset Taxonomy Report 2018'," 2018, Available: https://www.cryptocompare.com/media/34478555/cryptocomparecryptoasset-taxonomy-report-2018.pdf.

[31] M. ElMessiry and A. ElMessiry, "Blockchain Framework for Textile Supply Chain Management," in International Conference on Blockchain, 2018, pp. 213-227: Springer.

[32] Y. Hui, "What is a digital object?," Metaphilosophy, vol. 43, no. 4, pp. 380-395, 2012.

[33] A. Faget, "Fungible vs Non-Fungible Tokens: What's the Difference?," ed, 2018.

[34] P. S. A. S. Board, Definition and recognition of the elements of financial statements. Australian Accounting Research Foundation, 1992.

[35] W. Maas, "Classification and valuation issues for crypto-assets," ed, 2018.

[36] T. T. A. Dinh, J. Wang, G. Chen, R. Liu, B. C. Ooi, and K.-L. Tan, "Blockbench: A framework for analyzing private blockchains," in Proceedings of the 2017 ACM International Conference on Management of Data, 2017, pp. 1085-1100: ACM.

[37] G. Söderberg, "Are Bitcoin and other crypto-assets money?," Economic Commentaries, no. 5, p. 14, 2018.

[38] L. Deikun, "Explain me like I'm five: What is Cryptocurrency," ed, 2018.

[39] mybitcoin, "Blockchain Digital Asset Classification: Types Of Cryptocurrencies?," ed, 2018.

[40] M. Demertzis and G. B. Wolff, "The economic potential and risks of crypto assets: is a regulatory framework needed?," Bruegel Policy Contribution, no. 14, 2018.

[41] S. Huckle, R. Bhattacharya, M. White, and N. Beloff, "Internet of things, blockchain and shared economy applications," Procedia computer science, vol. 98, pp. 461-466, 2016.

[42] K.-L. Hui, A. Vance, and D. Zhdanov, "Securing digital assets," MIS Quarterly Research, 2018.

[43] A. Savelyev, "Copyright in the blockchain era: Promises and challenges," Computer law \& security review, vol. 34, no. 3, pp. 550-561, 2018.

[44] Katalyse.io. (2018). Security Tokens vs. Utility Tokens-How different are they? Available: https://hackernoon.com/security-tokens-vs-utility-tokens-how-differentare-they-22d6be8901c2

[45] M. Middelman, "Why utility tokens will not make you rich," ed, 2018.

[46] M. Rosenfeld, "Overview of colored coins," White paper, bitcoil. co. il, vol. 41, 2012. 